\title{
Continuous quasi-hyperbolic discounting
}

DOI:

10.1016/j.jmateco.2016.04.001

\section{Document Version}

Accepted author manuscript

Link to publication record in Manchester Research Explorer

\section{Citation for published version (APA):}

Webb, C. (2016). Continuous quasi-hyperbolic discounting. Journal of Mathematical Economics, 64, 99-106. https://doi.org/10.1016/j.jmateco.2016.04.001

\section{Published in:}

Journal of Mathematical Economics

\section{Citing this paper}

Please note that where the full-text provided on Manchester Research Explorer is the Author Accepted Manuscript or Proof version this may differ from the final Published version. If citing, it is advised that you check and use the publisher's definitive version.

\section{General rights}

Copyright and moral rights for the publications made accessible in the Research Explorer are retained by the authors and/or other copyright owners and it is a condition of accessing publications that users recognise and abide by the legal requirements associated with these rights.

\section{Takedown policy}

If you believe that this document breaches copyright please refer to the University of Manchester's Takedown Procedures [http://man.ac.uk/04Y6Bo] or contact uml.scholarlycommunications@manchester.ac.uk providing relevant details, so we can investigate your claim.

\section{OPEN ACCESS}




\title{
Continuous Quasi-Hyperbolic Discounting.
}

\author{
Craig S. Webb*
}

March 31, 2016

\begin{abstract}
This paper studies intertemporal choice in a dynamic framework with continuous time. A model called continuous quasi-hyperbolic discounting is considered, extending the popular quasi-hyperbolic discounting to an integral form. Dynamic preference axioms, time consistency and time invariance, are formulated and used to provide a foundation for an integral form of exponential discounting; the central model of dynamic, intertemporal choice in economics. A relaxion of the time consistency axiom, complementary time consistency, is formulated to provide a dynamic preference foundation for continuous quasi-hyperbolic discounting. A preference condition for present bias is also characterised in the context of the model.

Keywords: Exponential discounting; Quasi-hyperbolic discounting; Present bias; Time Consistency.

JEL Classification: D90.
\end{abstract}

*Address: Economics, School of Social Sciences, The University of Manchester, England. Contactcraig.webb@manchester.ac.uk.

${ }^{\dagger}$ I am grateful for the helpful comments of the editor and two anonymous reviewers. The usual disclaimer applies. 


\section{Introduction}

Under exponential discounting, the dominant paradigm for intertemporal choice in economics, future utility is discounted at a constant rate. Evidence suggests, however, that people often display present biased preferences (Thaler, 1981) -

contrary to a constant discount rate. Quasi-hyperbolic discounting (Phelps and Pollak, 1968) is a popular model of present biased preferences. Quasi-hyperbolic discounting is simple and tractable, and has been used in a variety of economic applications (Asheim, 1997; Laibson, 1997; Barro, 1999; Diamond and Koszegi, 2003; Luttmer and Mariotti, 2003). Although quasi-hyperbolic discounting was developed for discrete time, many economic applications involve consumption streams in continuous time and require an integral form of discounted utility.

In discrete time, axiomatisations of quasi-hyperbolic discounting have been presented by Hayashi (2003), Attema, Bleichrodt, Rohde and Wakker (2010), and Olea and Strzalecki (2014). There has been no previous axiomatisation of quasihyperbolic discounting in integral form. Indeed, it turns out that how to extend quasi-hyperbolic discounting to continuous time is not immediately obvious. There is more than one possible approach. To derive an integral representation axiomatically, the approach taken here considers a discount function due to Jamison and Jamison (2011). The resulting model is called continuous quasi-hyperbolic (CQH) discounting. $\mathrm{CQH}$ discounting retains the intuitive properties of discrete quasihyperbolic discounting, in a form more convenient for continuous time applications. Pan, Webb and Zank (2015) provided an axiomatic preference foundation for $\mathrm{CQH}$ discounting for the timed outcome framework, à la Fishburn and Rubinstein (1982). This paper provides an axiomatic foundation for the integral form of $\mathrm{CQH}$ discounting over consumption streams. Only the richness naturally provided by the time dimension is used, allowing the outcome set to be arbitrary. Hence, the model can be applied to monetary outcomes, health outcomes, durable goods, and so on. 
Despite its long history and central place in economics, a preference foundation for the integral form of exponential discounting in continuous time was only recently obtained by Kopylov (2010). ${ }^{1}$ As a special case of CQH discounting, this paper extends Kopylov's static preference foundation to a dynamic framework, providing, to the best of my knowledge, the first foundation for exponential discounting for continuous time based on the dynamic preference principles of time invariance and time consistency. Time consistency is an appealing property of exponential discounting. Because of its normative content, it is important to understand precisely how time consistency is violated by $\mathrm{CQH}$ discounting. This paper provides an axiom, called complementary time consistency, that weakens the time consistency axiom. Replacing the time consistency axiom, in dynamic exponential discounting, with the complementary time consistency axiom characterises $\mathrm{CQH}$ discounting.

The paper is organised as follows: Section 2 describes the notation used in the paper, Section 3 presents the model formally, Section 4 covers the basic axioms, Section 5 characterises the class of time invariant, additively and multiplicatively separable representations, Section 6 characterises exponential discounting, and Section 7 formulates the two-stage consistency axiom and characterises continuous quasi-hyperbolic discounting. In Section 8 a preference condition capturing present bias is formulated and characterised under $\mathrm{CQH}$ discounting. All proofs are in the appendix.

\section{Definitions}

Let $X$ be a set of outcomes and let time be $T=[0, \infty)$. The present, denoted $\eta$, is the time at which a decision maker makes a decision. Denote by $T_{\eta}$ the interval

\footnotetext{
${ }^{1}$ As noted by Harvey and Osterdal (2012:285), it is "surprising that integral discounting models were not developed long ago - and many readers may assume that they have been."
} 
$[\eta, \infty)$. Let $C_{\eta}$ denote the set of consumption streams, the set of step functions $x: T_{\eta} \rightarrow X$. That is, functions that are constant on intervals $\left[a_{i-1}, a_{i}\right)$ for some $\eta=a_{0}<a_{1}<\cdots<a_{n-1}<a_{n}=\infty$. Typical elements of $C_{\eta}$ are $x, y, z$. A consumption stream $x$ is a constant stream if, for all $s, t \in T_{\eta}, x(s)=x(t)$. The set of constant streams is $\mathcal{C}_{\eta}^{*}$. For consumption streams $x, y \in \mathcal{C}_{\eta}$ and $\eta \leqslant a \leqslant b$, the notation $x[a, b) y$ is used to denote the consumption stream with outcome $x(t)$ for all $t \in[a, b)$ and outcome $y(t)$ for all $t \notin[a, b)$.

At each $\eta$, the decision maker chooses so as to maximise their static, present preference relation $\succsim_{\eta} \subseteq \mathcal{C}_{\eta} \times \mathcal{C}_{\eta}$. For $x, y \in \mathcal{C}_{\eta}$, interpret $x \geqslant_{\eta} y$ as "stream $y$ is not preferred to stream $x$ at decision time $\eta$." If $x, y \in \mathcal{C}_{0}, x \geqslant_{\eta} y$ means that $\left.x\right|_{T_{\eta}} \geqslant\left._{\eta} y\right|_{T_{\eta}}$, where $\left.x\right|_{T_{\eta}}$ and $\left.y\right|_{T_{\eta}}$ are the respective restrictions of $x$ and $y$ to $T_{\eta}$. Preferences for outcomes are derived from preferences for constant streams. That is, for $x, y \in C_{\eta}$ write $x(t) \geqslant_{\eta} y(t)$ if the constant stream $\tilde{y} \in C_{\eta}^{*}$ always equal to outcome $y(t)$ is not preferred to the constant stream $\tilde{x} \in \mathcal{C}_{\eta}^{*}$ always equal to outcome $x(t)$.

A dynamic preference structure is a collection of static preference relations $\mathcal{R}=$ $\left\{\gtrsim_{\eta}\right\}_{\eta \in T}$. A dynamic model $\mathcal{V}=\left\{V_{\eta}\right\}_{\eta \in T}$ is a collection of real-valued functions $V_{\eta}: \mathcal{C}_{\eta} \rightarrow \mathbb{R}$. A dynamic preference structure $\mathcal{R}$ is represented by a dynamic model $\mathcal{V}$ if for each $\gtrsim_{\eta} \in \mathcal{R}$ there is a $V_{\eta} \in \mathcal{V}$ such that, for all $x, y \in \mathcal{C}_{\eta}, x \gtrsim_{\eta} y$ if and only if $V_{\eta}(x) \geqslant V_{\eta}(y)$.

Invariant separable discounting holds if $\mathcal{R}$ is represented by a dynamic model $\mathcal{V}$ such that, for all $V_{\eta} \in \mathcal{V}$ :

$$
V_{\eta}(x)=\int_{\eta}^{\infty} D(t-\eta) u(x(t)) d t .
$$

with $u: X \rightarrow \mathbb{R}$ a $\gtrsim_{\eta}$-increasing utility function for outcomes, and $D: T_{\eta} \rightarrow$ $\mathbb{R}$ a strictly decreasing and continuous discount function, with $D(0)=1$ and $\lim _{t \rightarrow \infty} D(t-\eta)=0$. Exponential discounting holds if $\mathscr{R}$ is represented by a 
dynamic model $\mathscr{V}$ such that, for all $V_{\eta} \in \mathscr{V}$ :

$$
V_{\eta}(x)=\int_{\eta}^{\infty} \delta^{t-\eta} u(x(t)) d t .
$$

with $u: X \rightarrow \mathbb{R}$ a $\geqslant_{\eta}$-increasing utility function for outcomes, and $\delta \in(0,1)$ the discount factor.

\section{Continuous Quasi-Hyperbolic Discounting}

In certain applications, time is taken to be discrete. For example taking $T=$ $\{0,1,2, \ldots\}$. Instead of consumption streams, the objects of choice in the discrete time framework are called consumption sequences. A sequence $x$ gives outcome $x(t)$ at time $t \in\{0,1,2, \ldots\}$. Discrete quasi-hyperbolic discounting holds if $\mathscr{R}$, restricted to sequences of outcomes, is represented by $\mathscr{V}$ such that, for all $V_{\eta} \in \mathscr{V}$ :

$$
V_{\eta}(x)=u(x(\eta))+\beta \sum_{t=\eta+1}^{\infty} \delta^{t-\eta} u(x(t)),
$$

with $u: X \rightarrow \mathbb{R} a \geqslant_{\eta}$-increasing utility function for outcomes, $\delta \in(0,1)$ the discount factor, and $\beta>0$ the penalty factor. If $\beta<1$, then outcomes occuring after the immediate present $\eta$ are penalised an amount in addition to the discount factor, thus capturing present-biased preferences. This section considers the extension of discrete quasi-hyperbolic discounting to continuous time.

Discrete quasi-hyperbolic discounting corresponds to invariant separable discounting with a discount function which, at times $t-\eta=0,1,2, \ldots$, gives $1, \beta \delta, \beta \delta^{2}, \ldots$. One discount function in continuous time that agrees with discrete quasi-hyperbolic discounting, and has also been called the quasi-hyperbolic discount function, is the following:

$$
D(t-\eta)=\left\{\begin{array}{cc}
1 & \text { if } t-\eta=0, \\
\beta \delta^{t-\eta} & \text { if } t-\eta>0 .
\end{array}\right.
$$


with $0 \leqslant \delta \leqslant 1$ and $0 \leqslant \beta \leqslant 1$. Harris and Laibson (2013) referred to invariant separable discounting with discount function (1) as the instantaneous gratification model. It arises as a limiting case of the following discount function:

$$
D(t-\eta)=\left\{\begin{array}{cc}
\delta^{t-\eta} & \text { if } t-\eta<\lambda, \\
\beta \delta^{t-\eta} & \text { if } t-\eta \geqslant \lambda .
\end{array}\right.
$$

with $0 \leqslant \delta \leqslant 1,0 \leqslant \beta \leqslant 1$, and $0<\lambda<\infty$. Under this discount function, delays shorter than and longer than $\lambda$ are discounted by the factor same $\delta$, but the penalty term $\beta$ is applied only to longer delays. Discount function (1) is the limiting case as $\lambda \rightarrow 0$. Harris and Laibson (2013) demonstrate how this discount function can be successfully applied to a consumption-savings model. The above discount function, however, is problematic in the continuous time framework. It is continuous if and only if $\beta=1$. For an integral representation, it is more convenient to assume a continuous discount function. ${ }^{2}$ Consider, instead, the following continuous discount function, due to Jamison and Jamison (2011):

$$
D(t-\eta)=\left\{\begin{array}{cc}
\left(\beta^{\frac{1}{\lambda}} \delta\right)^{t-\eta} & \text { if } t-\eta<\lambda, \\
\beta \delta^{t-\eta} & \text { if } t-\eta \geqslant \lambda .
\end{array}\right.
$$

with $0<\lambda<\infty, 0 \leqslant \delta \leqslant 1$, and $0 \leqslant \beta \leqslant \frac{1}{\delta^{\lambda}}$. The present, instead of being a single point $\eta$, is an interval $[\eta, \lambda]$, where $\lambda$ is a subjective parameter, the switch point, that delineates the present from the future. Delays shorter than $\lambda$ are discounted exponentially using the discount factor $\left(\beta^{\frac{1}{\lambda}} \delta\right)$, and delays longer than $\lambda$ are weighted by a penalty factor $\beta$ and discounted exponentially using the discount factor $\delta$. Discount function (3) is continuous everywhere, in particular:

$$
\lim _{s \rightarrow \lambda^{-}}\left(\beta^{\frac{1}{\lambda}} \delta\right)^{s}=\lim _{s \rightarrow \lambda^{+}} \beta \delta^{s}=\beta \delta^{\lambda} .
$$

\footnotetext{
${ }^{2}$ In their application of the instantaneous gratification model, Harris and Laibson (2013) smoothed out this problem by assuming $\gamma$ is stochastic with a known exponential distribution, hence the model becomes continuous in expectation. In this paper, we consider deterministic preferences.
} 
If $\lambda \in(0,1)$, then the above discount function at times $t-\eta=0,1,2, \ldots$ gives $1, \beta \delta, \beta \delta^{2}, \ldots$, agreeing with discrete quasi-hyperbolic discounting. Because of this, we call the special case of invariant separable discounting, with discount function (3), continuous quasi-hyperbolic $(\mathrm{CQH})$ discounting. That is, $\mathrm{CQH}$ discounting holds if $\mathscr{R}$ is represented by $\mathscr{V}$ such that, for all $V_{\eta} \in \mathscr{V}$ :

$$
V_{\eta}(x)=\int_{\eta}^{\eta+\lambda}\left(\beta^{\frac{1}{\lambda}} \delta\right)^{t-\eta} u(x(t)) d t+\beta \int_{\eta+\lambda}^{\infty} \delta^{t-\eta} u(x(t)) d t,
$$

with $0<\lambda<\infty, 0 \leqslant \delta \leqslant 1,0 \leqslant \beta \leqslant \frac{1}{\delta^{\lambda}}$, and $u: X \rightarrow \mathbb{R}$ a $\geqslant_{\eta}$-increasing utility function for outcomes. Exponential discounting is the special case of $\mathrm{CQH}$ discounting with $\beta=1$. $\mathrm{CQH}$ discounting retains the intuitive properties of discrete quasi-hyperbolic discounting, in a form more convenient for continuous time applications. The main theorem of this paper, presented in Section 7, provides a dynamic preference foundation for $\mathrm{CQH}$ discounting over consumption streams.

Under $\mathrm{CQH}$ discounting, the switch point is a subjective parameter, expressed in terms of delay from the decision time. For example, if the switch point is one month from the decision time, then utility in six months is discounted using the penalty factor $\beta$ and discount factor $\delta$. Five and half months later, utility at the same point in calendar time would be less than one month from the decision time. At this decision time, the same utility would be classed as "in the present" and discounted using the, possibly different, discount factor $\left(\beta^{\frac{1}{\lambda}} \delta\right)$. In this way, $\mathrm{CQH}$ discounting is able to account for the typical change their plans associated with procrastination. $\mathrm{CQH}$ discounting, in general, allows for decreasing or increasing impatience. In section 8 a preference condition for decreasing impatience leading to present bias is formulated which, under $\mathrm{CQH}$ discounting, is equivalent to $\beta \leqslant 1$. CQH discounting (3) and Harris and Laibson's discount function (2) both belong to the general class of semi-hyperbolic discount functions, characterised by Olea 
and Strzalecki (2014):

$$
D(t-\eta)=\left\{\begin{array}{cc}
D(t-\eta) & \text { if } t-\eta<\lambda, \\
\beta \delta^{t-\eta} & \text { if } t-\eta \geqslant \lambda .
\end{array}\right.
$$

with $D$ strictly decreasing everywhere. Semi-hyperbolic discounting does not constrain the discount function for delays shorter than $\lambda$, then takes the exponential form for all longer delays. Note that, in their axiomatisation of (5), Olea and Strzalecki (2014) assumed $\lambda$ as a primitive. In the preference foundation for $\mathrm{CQH}$ discounting, $\lambda$ is derived endogenously from preferences.

\section{Basic Axioms}

The following five axioms are assumed:

Axiom 1 (Weak Ordering): For all $\succsim_{\eta} \in \mathcal{R}, \succsim_{\eta}$ over $\mathcal{C}_{\eta}$ is complete and transitive. Axiom 2 (Common Outcome Independence): For all $\gtrsim_{\eta} \in \mathcal{R}, x, y, z, \tilde{z} \in \mathcal{C}_{\eta}$, and $a \leqslant b$ :

$$
x[a, b) z \gtrsim_{\eta} y[a, b) z \Rightarrow x[a, b) \tilde{z} \gtrsim_{\eta} y[a, b) \tilde{z} .
$$

Axiom 3 (Interval Monotonicity): For all $\gtrsim_{\eta} \in \mathcal{R}, x, y \in \mathcal{C}_{\eta}$, if $x(t) \gtrsim_{\eta} y(t)$ for all $t \in T_{\eta}$, then $x \gtrsim_{\eta} y$. If it also holds that $x(t)>_{\eta} y(t)$ for all $t \in[a, b)$, for some $a<b$, then $x>_{\eta} y$.

Axiom 4 (Weak outcome separability): For all $\gtrsim_{\eta} \in \mathcal{R}, a \leqslant b, c \leqslant d$, and all $x, y, \tilde{x}, \tilde{y} \in \mathcal{C}_{\eta}^{*}$, with $x>_{\eta} y$ and $\tilde{x}>_{\eta} \tilde{y}$ :

$$
x[a, b) y>_{\eta} x[c, d) y \Rightarrow \tilde{x}[a, b) \tilde{y}>_{\eta} \tilde{x}[c, d) \tilde{y} .
$$

Axiom 5 (Strong Monotone Continuity): For all $\gtrsim_{\eta} \in \mathcal{R}, x \in \mathcal{C}_{\eta}^{*}, y, z \in \mathcal{C}_{\eta}$, and $\left\{\left[a_{i}, b_{i}\right)\right\}_{i=1}^{\infty}$ with $\left[a_{1}, b_{1}\right] \supset\left[a_{2}, b_{2}\right] \supset \ldots$ and $\cap_{i=1}^{\infty}\left[a_{i}, b_{i}\right)$ either empty or a single point, if $y \succsim_{\eta} x\left[a_{i}, b_{i}\right) z$ for all $i$, or if $x\left[a_{i}, b_{i}\right) y \succsim_{\eta} z$ for all $i$, then $y \succsim_{\eta} z$. 
Weak ordering is necessary for a dynamic preference structure $\mathcal{R}$ to admit a dynamic model representation $\mathcal{V}$. Common outcome independence, formally equivalent to axiom P2 of Savage (1954), is necessary for additive separability of each $V_{\eta} \in \mathcal{V}$. Interval monotonicity is a standard monotonicity assumption, except that strict preference is required when one stream dominates another and strictly dominates on a non-degenerate interval. Weak outcome separability, formally equivalent to axiom P4 of Savage (1954), is necessary for multiplicative separability of time discounting from outcome utility. Strong monotone continuity, introduced by Kopylov (2010), adapts the monotone continuity axioms used by Villegas (1964) and Arrow (1970).

\section{Time Invariance}

Given a consumption stream $x \in \mathcal{C}_{0}$ and $\eta \in T$, denote by $x_{\eta}$ the stream $x_{\eta} \in \mathcal{C}_{\eta}$ such that $x_{\eta}(t)=x(t-\eta)$ for all $t \in T_{\eta}$. Although a stream $x \in \mathcal{C}_{0}$ and the stream $x_{\eta}$ are different objects, there is a particular lens through which they appear identical. At time zero, under stream $x$, the decision maker waits $t$ units of time to receive outcome $x(t)$. At decision time $\eta$, under stream $x_{\eta}$, the decision maker also waits $t$ units to receive outcome $x_{\eta}(\eta+t)=x(t)$. Hence, if a decision maker considers consumption streams only in terms of delay, relative to decision time, these streams appear identical. The behavioural implications of this perspective, called time invariance, are formalised in terms of a relationship between preference relations in the dynamic preference structure as follows:

Axiom 6 (Time Invariance): For all $x, y \in \mathcal{C}_{0}, x \gtrsim_{0} y$ if and only if $x_{\eta} \gtrsim_{\eta} y_{\eta}$.

When combined with the basic axioms, time invariance is necessary and sufficient for invariant separable discounting:

Theorem 5.1. The dynamic preference structure $\mathcal{R}$ satisfies axioms 1-6 (weak ordering, common outcome independence, interval monotonicity, weak outcome sep- 


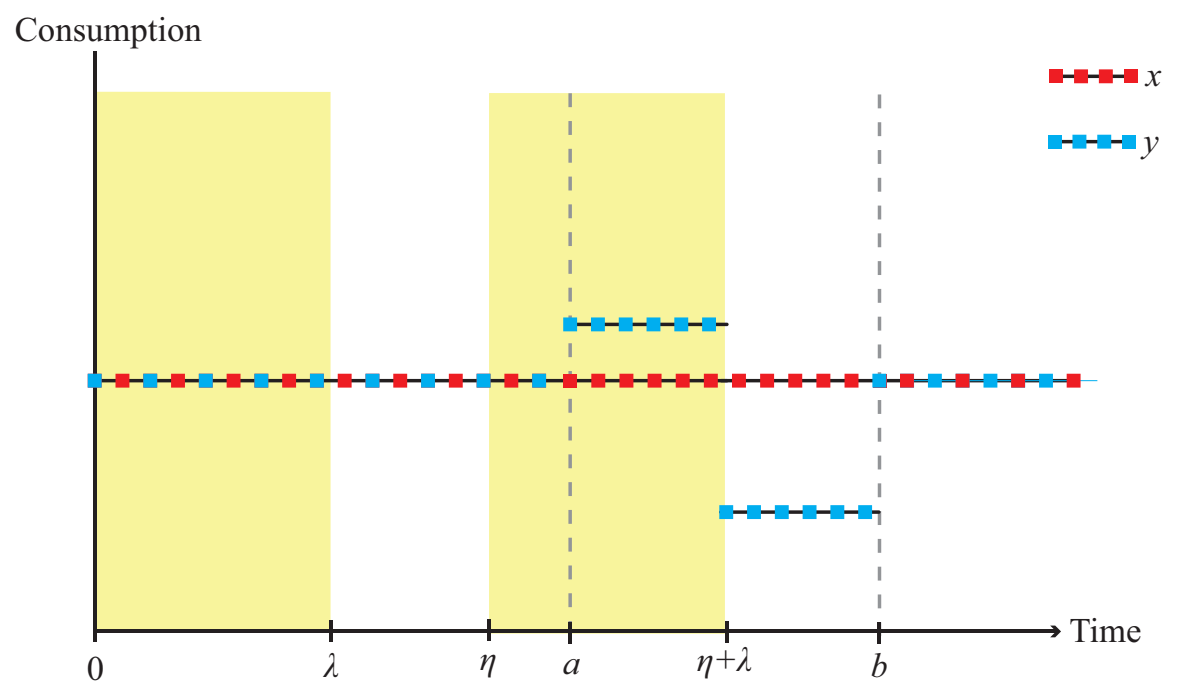

Figure 1: Continuous quasi-hyperbolic discounting and violations of time consistency, which requires $x \gtrsim_{0} y$ if and only if $x \succsim_{\eta} y$.

arability, strong monotone continuity, and time invariance) if and only if invariant separable discounting holds. The discount function $D$ is uniquely determined and utility for outcomes u is unique up to positive affine transformation.

Theorem 5.1 is proved in Appendix A.2.

\section{Time Consistency}

An invariant separable discounter will, inevitably, repeat the same behaviour with each new decision time. Suppose that, today, such a decision maker plans to start saving in six months time. Then, six months later, the decision maker will again prefer to wait six months to start saving. The decision maker procrastinates. In terms of observed behaviour, the decision maker appears consistent; repeating the same pattern of not saving each day. However, there is an inconsistency if one compares observed with planned behaviour. Time consistency refers to consistency of 
planned behaviour with observed behaviour. In this section, a preference-based definition of time consistency is formulated.

Consider the streams $x$ and $y$ in Figure 1. Choosing stream $y$ over $x$ will involve a period of increased consumption followed a period of decreased consumption. Suppose that, at time zero, the decision maker prefers $x$ to $y$. Clearly, if the decision is reconsidered at time $\eta+\lambda$, the beneficial part of $y$ has passed, and the decision maker would do well to choose $x$, regardless of their preference at earlier times. Before time $a$, however, the differences between $x$ and $y$ are yet to materialise. Reversing one's earlier preference at any of those times is the behaviour ruled out by the following axiom:

Axiom 7 (Time Consistency): For all $x, y, z \in \mathcal{C}_{0}$ and $\eta \in T$, if $x=x[a, b) z$ and $y=y[a, b) z$ for some $\eta \leqslant a \leqslant b$, then $x \gtrsim_{0} y$ if and only if $x \gtrsim_{\eta} y$.

Consider two streams that are identical, except on some interval $[a, b)$. Time consistency requires that, at any time before this interval, the decision maker does not reverse previously expressed preferences. We have the following theorem:

Theorem 6.1. If invariant separable discounting holds, $\mathcal{R}$ satisfies time consistency if and only if exponential discounting holds. The discount factor $\delta$ is uniquely determined and utility for outcomes $u$ is unique up to positive affine transformation.

The proof of Theorem 6.1 will follow as a corollary of the paper's main theorem, presented in the next section. Note that a similar theorem, in the timed outcome framework, has been obtained by Halevy (2015).

Consider again Figure 1. Suppose that, at time zero, a continuous quasi-hyperbolic $(\mathrm{CQH})$ discounter prefers $x$ to $y$. When considered at time zero, the relative costs and benefits of choosing $y$ occur more than $\lambda$ time units from the decision time, hence they are evaluated using the same discount factor. At time $\eta$, the preference may be different. The relative costs of choosing $y$ occur after $\eta+\lambda$ and can, therefore, be discounted differently than the relative benefits of $y$, which occur almost 
immediately after $\eta$. The possibility of reversing one's preference arises, therefore $\mathrm{CQH}$ discounters can violate of time consistency.

\section{Complementary Time Consistency}

Since Thaler (1980), there has been a great deal of evidence to suggest that decision makers' preferences often exhibit present bias. For example, decision makers who today prefer $£ 120$ in 13 months to $£ 100$ in 12 months, will often prefer $£ 100$ today to $£ 120$ in one month. In both cases the decision maker must wait one month for an additional $£ 20$. Such a delay is considered acceptable, if it occurs in the future, but is disliked when money is immediately available.

If time invariance is assumed, present bias preferences lead to violations of time consistency. Under time invariance, the initial preference for $£ 100$ today over $£ 120$ in one month means that the initial preference for $£ 120$ in 13 months over $£ 100$ in 12 months will be reversed if the choice is reconsidered in 12 months. CQH discounting preferences are compatible with present bias, and its converse future bias, hence can violate time consistency. We use the term present affected to mean either present bias or future bias. This section considers how the time consistency axiom can be relaxed to allow for present affected preferences. Furthermore, we allow the terms "present" and "after the present" to be subjectively defined.

Present affected preferences manifest themselves only when comparing delays in the present with delays after the present. Allowing for present affected preferences does not, therefore, require that the time consistency axiom is amended for decisions involving streams that differ only in the sufficiently remote future. To formalise this, consider the following:

Definition 7.1. Time-consistency-beyond-t-from-now: For all $x, y, z \in \mathcal{C}_{0}$ and $\eta \in T$, if $x=x[a, b) z$ and $y=y[a, b) z$ for some $\eta \leqslant t \leqslant a \leqslant b$, then $x \gtrsim_{\eta} y$ only if $x \gtrsim_{0} y$. 
Provided that two streams do not differ before time $t$, a dynamic preference structure $\mathcal{R}$ that satisfying the above condition behaves consistently. Such a condition seems reasonable when $t$ is far enough in the future to be considered "after the present". ${ }^{3}$ Consider also the following analogous condition:

Definition 7.2. Time-consistency-within-t-from-now: For all $x, y, z \in \mathcal{C}_{0}$ and $\eta \in T$, if $x=x[a, b) z$ and $y=y[a, b) z$ for some $\eta \leqslant a \leqslant b \leqslant t$, then $x \gtrsim_{0} y$ only if $x \gtrsim_{\eta} y$.

The above condition seems reasonable when $t$ is sufficiently close to the decision time, such that the streams above differ only in the "present".

We can now formulate our axiom, complementary time consistency. To focus on present effects alone, we consider preferences that may be present affected, but are otherwise time consistent. In this case, a violation of time-consistency-within$t$-from-now occurs only when the time interval from now to $t$ contains both the "present" period and an "after the present" period. Then, it must be that the time interval after $t$ contains only "after the present" times. As such, whenever timeconsistency-within- $t$-from-now is violated, we must exclude violations of timeconsistency-beyond- $t$-from-now. Analogously, a violation of time-consistencybeyond- $t$-from-now occurs only when the time interval after $t$ contains part of the "present" period and an "after the present" period. In this case, the time interval before $t$ must contain only "present" times, hence we must exclude violations of time-consistency-within-t-from-now. This is summarised in the following axiom: Axiom 7* (Complementary Time Consistency): For all $t \in T, \mathcal{R}$ satisfies at least one of time-consistency-within-t-from-now or time-consistency-beyond$t$-from-now.

\footnotetext{
${ }^{3}$ This formalises the intuition of Strotz (1956:179), who wrote "I would have confidence in the judiciousness of a person today, if he is not ignorant of future facts, to decide how much to save and how much to spend for the rest of his life, starting a couple of years from now" (emphasis in the original).
} 
To understand why complementary time consistency is necessary for CQH discounting, first consider the following:

Lemma 7.3. Continuous quasi-hyperbolic discounting holds, with switch point $\lambda$, only if $\mathcal{R}$ satisfies time-consistency-within- $\lambda$-from-now and time-consistencybeyond- $\lambda$-from-now.

Suppose that, at a given time $t$, we observe a CQH discounter violate time-consistencywithin-t-from-now. From such an observation, we may infer that the switch point has been crossed, hence $\lambda<t$. The complementary condition, time-consistencybeyond-t-from-now, then must hold. A violation of the latter condition would imply $\lambda>t$, which has been ruled out. Conversely, an observed violation of time-consistency-beyond- $t$-from-now must entail that the complementary condition, time-consistency-within- $t$-from-now, holds. Hence, complementary time consistency is necessary for CQH discounting.

In certain circumstances, there may be a natural candidate for $\lambda$ the switch point. In such cases, it may be reasonable to take $\lambda$ as a primitive, and postulate timeconsistency-within- $\lambda$-from-now and time-consistency-beyond- $\lambda$-from-now as axioms. This would deliver $\mathrm{CQH}$ discounting. In general, however, it is desirable to derive the switch point $\lambda$ from preferences. It will be shown that the complementary time consistency axiom delivers, from preferences, both the switch point and the consistency properties associated with the derived switch point. The main theorem of this paper can now be stated:

Theorem 7.4. If invariant separable discounting holds, the dynamic preference structure $\mathcal{R}$ satisfies complementary time consistency if and only if continuous quasi-hyperbolic discounting holds. The parameters are uniquely determined when meaningful.

Theorem 7.4 is proved in Appendix A.2. It can be shown that $\mathcal{R}$ satisfies either time-consistency-within- $t$-from-now for all $t \in T$, or time-consistency-beyond- $t$ - 
from-now for all $t \in T$, if and only if $\mathcal{R}$ satisfies time consistency. Under $\mathrm{CQH}$ discounting, time consistency follows if and only if $\beta=1$, hence Theorem 6.1 follows as a corollary of Theorem 7.4.

\section{Subjective Present Bias}

In the previous section, we defined present affected preferences as preferences exhibiting either present bias or future bias. There is evidence supporting both types of bias, and CQH discounting is compatible with either type, but the overwhelming majority of present affected decision makers seem to be present biased. It is, therefore, interesting to characterise the class of $\mathrm{CQH}$ discounters exhibiting present bias. This corresponds to the case where delays after the present seem to be penalised less severely than delays in the present. Under CQH discounting, this requires that the initial discount factor $\left(\beta^{\frac{1}{\lambda}} \delta\right)$ is less than the future discount factor $\delta$, which is equivalent to $\beta<1$. In this section, a preference axiom is formulated called subjective present bias that characterises this condition under $\mathrm{CQH}$ discounting. ${ }^{4}$

Recall the following axiom, formulated in this framework by Kopylov (2010):

Axiom 8 (Stationarity): For all $x, y \in \mathcal{C}_{0}^{*}, 0<a<b<c<d<\infty$ and $t>0$, $x[a, b) y \sim_{0} x[c, d) y$ only if $x[a+t, b+t) y \sim_{0} x[c+t, d+t) y$.

Under exponential discounting, utility is discounted by a constant factor over time. The stationarity axiom, which is necessary for exponential discounting, expresses this constant level of impatience as a preference condition. When comparing streams that differ on intervals $[a, b)$ and $[c, d)$, stationarity asserts that indifferences are not upset when these intervals are delayed by a common delay $t>0$. If stationarity is assumed, $\mathrm{CQH}$ discounting reduces to exponential discounting.

\footnotetext{
${ }^{4}$ The analysis of this section, and much of the proof of Proposition 8.1, were suggested by an anonymous reviewer.
} 
Present bias is a direct violation of the stationarity axiom. In this paper, we allow the period called the "present" to be subjectively defined. Our definition of "present" is, essentially, the period that a present-biased decision maker is biased towards. ${ }^{5}$ To operationalise this idea, suppose that a violation of stationarity is observed:

$$
x[a, b) y \sim_{0} x[c, d) y \quad \text { and } \quad x[a+t, b+t) y \nsim_{0} x[c+t, d+t) y, \quad \text { with } x>_{0} y .
$$

If present bias is driving the above violation of stationarity, it cannot be that the $x$ outcome in each stream occurs only in the present. Hence, the present period must end no later than time $d+t$. Similarly, it cannot be that the $x$ outcome in each stream above occurs only after the present, hence the present period must not end before the earliest time $a$. The above preferences, therefore, suggest that the threshold ending the present period lies in the interval $[a, d+t]$.

Suppose that we also observe the indifference:

$$
x[\tilde{a}, \tilde{b}) y \sim_{0} x[\tilde{c}, \tilde{d}) y, \quad \text { where } \tilde{a}<\tilde{b}<a \text { and } d+t<\tilde{c}<\tilde{d} .
$$

Having established that, for this decision maker, the threshold ending the present period lies in the interval $[a, d+t]$, it is apparent that the interval $[\tilde{a}, \tilde{b})$ occurs entirely in the present and the interval $[\tilde{c}, \tilde{d})$ occurs entirely after the present. If a delay $s>0$ is now introduced, with $\tilde{b}+s<a$ so that this separation remains, then the decision maker is comparing a delay in the present with a delay after the present. For present biased preferences, the delay after the present is penalised less severely, hence one expects:

$$
x[\tilde{a}+s, \tilde{b}+s) y \prec_{0} x[\tilde{c}+s, \tilde{d}+s) y .
$$

This is formulated as an axiom:

\footnotetext{
${ }^{5}$ On first reading, this definition can seem somewhat circular. However, in their seminal paper on risk aversion, Rothschild and Stiglitz (1976) defined "risk" similarly. Machina and Rothschild (2008: 193) paraphrased their definition as "risk is what risk-averters hate."
} 
Axiom 8* (Subjective Present Bias): For all $x, y \in \mathcal{C}_{0}^{*}$ with $x>_{0} y, 0<a<b<$ $c<d<\infty$ and $t>0$, if $x[a, b) y \sim_{0} x[c, d) y$ and $x[a+t, b+t) y \nsim_{0} x[c+t, d+t) y$, then for all $0<\tilde{a}<\tilde{b}<\tilde{b}+s<a<d+t<\tilde{c}<\tilde{d}<\infty$, we have $x[\tilde{a}, \tilde{b}) y \sim_{0}$ $x[\tilde{c}, \tilde{d}) y$ only if $x[\tilde{a}+s, \tilde{b}+s) y \prec_{0} x[\tilde{c}+s, \tilde{d}+s) y$.

The following proposition characterises the implication of the subjective present bias axiom under continuous quasi-hyperbolic discounting:

Proposition 8.1. If continuous quasi-hyperbolic discounting holds, then the dynamic preference structure satisfies present bias if and only if $\beta \leqslant 1$.

The proof of Proposition 8.1 is presented in Appendix A.4.

\section{Discussion}

The choice of the dynamic framework in this paper warrants explanation. Instead of positing a static preference relation, a preference relation at each point in time was assumed. The key axioms, time invariance, time consistency and complementary time consistency, are dynamic axioms. They do not constrain preferences at a single point in time, but instead constrain how preferences change (or do not change) over time. ${ }^{6}$ An advantage of the framework used here is that it highlights the especially attractive features of exponential discounting, and precisely how $\mathrm{CQH}$ discounting deviates. Compare the requirements of stationarity (one's instantaneous preferences are invariant under translations that preserve relative delays) with time consistency (one should not reverse one's preferences for what are, in an absolute sense, the same objects) and time invariance (one should not reverse one's preferences for what are, in a relative sense, the same objects). The latter conditions are more suggestive of prescriptive principles to guide economic agents,

\footnotetext{
${ }^{6}$ Weak ordering, for example, could be called a static axiom in that it constrains preferences only at a fixed decision time.
} 
because avoiding preference reversals for the same objects provides a degree of immunity against economic ruin. Although Samuelson (1937) did not endorse exponential discounting as a normative model, he noted time consistency as an appealing property. ${ }^{7}$ In the context of choice under risk, Machina $(1989 ; 1685)$ argued that dynamic arguments prescribing expected utility were more "formidable" than those referring to static decisions. Those arguments also apply to choice over time with little translation.

It is not clear how to formulate preference axioms for invariant separable discounting assuming discount function (2). For $\mathrm{CQH}$ discounting, a falsifiable axiom has been formulated such that the switch point, where the discount factor changes, arises endogeneously from preferences. Under discount function (2), however, the switch point corresponds to a discontinuity. Such preferences, therefore, do not satisfy the strong monotone continuity axiom. Continuity axioms, in general, are not falsfiable. They are technical axioms, in the sense that either existential qualifiers or infinite sequences are used. Adapting the continuity axiom to allow for a discontinuity at a point determined engoneously by preferences could be possible. However, is not clear how to provide an axiom set that cleanly separates the behavioural from the technical in the way that Theorem 7.4 does for CQH discounting. Also note that, while it may be possible to adapt the techniques here to derive the continuous class of semi-hyperbolic discount functions (5), allowing the switch point to arise endogenously from preferences, this problem also remains open.

\footnotetext{
${ }^{7} \mathrm{He}$ writes, "The particular results we have reached are not subject to criticism on this score, having been carefully selected to take care of this provision. Contemplation of our particular equations will reveal that the results are unchanged even if the individual always discounts from the existing point of time rather than from the beginning of the period" (Samuelson, 1937; 160).
} 


\section{Closing Comments}

In this paper, quasi-hyperbolic discounting, which is popular for discrete time applications, has been extended to continuous time. An integral form of continuous quasi-hyperbolic has been characterised, based on a dynamic axiom called complementary time consistency. Complementary time consistency is a falsifiable axiom and, as such, calls for empirical tests. The experimental design of Halevy (2015) could be adapted for such a purpose. As with the discrete case, I expect that continuous quasi-hyperbolic discounting is at best an approximation to more realistic behaviour, but remains useful for other reasons. The main appeal of this model is its tractability, and this will manifest in future theoretical applications.

\section{References}

[1] Asheim, G. B. (1997). Individual and collective time-consistency. Rev Econ Stud 64 (1997) 427-443.

[2] Aczel, J. and F. Skof (2007). Local pexider and cauchy equations. Aequationes Mathematicae 73, 311-320.

[3] Arrow, K. (1970). Essays in the theory of risk-bearing. North-Holland.

[4] Barro, R. (1999). Laibson meets Ramsey in the neoclassical growth model. QJE 114, 1125-1152.

[5] Blackorby, C., D. Nissen, D. Primont and R. R. Russell (1973). Intertemporal decision making. Rev Econ Stud 40:2, 239-248.

[6] Diamond, P. and B. Koszegi (2003). Quasi-hyperbolic discounting and retirement. J Public Econ 87, 1839-1872. 
[7] Fishburn, P. C. and A. Rubinstein (1982). Time preference. Int Econ Rev 23, 677-694

[8] Halevy, Y. (2015). Time consistency: stationarity and time invariance. Econometrica 83:1, 335-352.

[9] Harris, C. and D. Laibson (2013). Instantaneous gratification. QJE, 128:1, 205-248.

[10] Hayashi, T. (2003). Quasi-stationary cardinal utility and present bias. J Econ Theory 112, 343-352.

[11] Harvey, C. M. and L. P. Osterdal (2012). Discounting models for outcomes over continuous time. J Math Econ 48, 284-294.

[12] Jamison, D. T. and J. Jamison (2011). Characterizing the amount and speed of discounting procedures. J Benefit Cost Anal 2:2, Article 1.

[13] Kopylov, I. (2010). Simple axioms for countably additive subjective probability. J Math Econ 46, 867-876.

[14] Laibson, D. (1997). Golden eggs and hyperbolic discounting. QJE 112:2, 443-477.

[15] Luttmer, E., and T. Mariotti (2003). Subjective discounting in an exchange economy. J Polit Econ 111:5, 959-989.

[16] Machina, M. J. (1989). Dynamic consistency and non-expected utility models of choice under uncertainty. J Econ Lit 27, 1622-1668.

[17] Machina, M. J. and M. Rothschild (2008). Risk. In The New Palgrave Dictionary of Economics, 2nd ed. Edited by S. N. Durlauf and L. E. Blume. Macmillan, London. 
[18] Olea, J. L. M. and T. Strzalecki (2014). Axiomatization and measurement of quasi-hyperbolic discounting. Quart J Econ 116, 121-160.

[19] Pan, J., C. S. Webb and H. Zank (2015). An extension of quasi-hyperbolic discounting to continuous time. Games Econ Behav 89, 43-55.

[20] Phelps, E. and R. Pollak (1968). On second-best national saving and gameequilibrium growth. Rev Econ Stud 35:2, 185-199.

[21] Rothschild, M. and J. E. Stiglitz (1970). Increasing risk I: A definition. J Econ Theory 2, 225-243.

[22] Samuelson, Paul (1937). A note on the measurement of utility. Rev Econ Stud $4: 2,155-161$.

[23] Savage, L. J. (1954). The foundation of statistics. Wiley, New York.

[24] Strotz, R. (1956). Myopia and inconsistency in dynamic utility maximization. Rev Econ Stud 23, 165-180.

[25] Thaler, R. H. (1981). Some empirical evidence of dynamic inconsistency. Econ Let 8, 201-207.

[26] Villegas, C. (1964). On qualitative probability $\sigma$-algebras. Ann Math Stat 35, 1787-1796.

\section{A Appendices}

\section{A.1 Proof of Lemma 7.3}

Let continuous quasi-hyperbolic discounting hold. It is shown first that timeconsistency-within- $\lambda$-from-now holds. The first preference $x[a, b) z \gtrsim_{0} y[a, b) z$, 
given $\eta \leqslant a \leqslant b \leqslant \lambda$, is equivalent to:

$$
\begin{gathered}
\int_{0}^{a}\left(\beta^{\frac{1}{\lambda}} \delta\right)^{t} u(z(t)) d t+\int_{a}^{b}\left(\beta^{\frac{1}{\lambda}} \delta\right)^{t} u(x(t)) d t+\int_{b}^{\lambda}\left(\beta^{\frac{1}{\lambda}} \delta\right)^{t} u(z(t)) d t+\beta \int_{\lambda}^{\infty} \delta^{t} u(z(t)) d t \\
\geqslant \\
\int_{0}^{a}\left(\beta^{\frac{1}{\lambda}} \delta\right)^{t} u(z(t)) d t+\int_{a}^{b}\left(\beta^{\frac{1}{\lambda}} \delta\right)^{t} u(y(t)) d t+\int_{b}^{\lambda}\left(\beta^{\frac{1}{\lambda}} \delta\right)^{t} u(z(t)) d t+\beta \int_{\lambda}^{\infty} \delta^{t} u(z(t)) d t
\end{gathered}
$$

which holds if and only if $\int_{a}^{b}\left(\beta^{\frac{1}{\lambda}} \delta\right)^{t} u(x(t)) d t \geqslant \int_{a}^{b}\left(\beta^{\frac{1}{\lambda}} \delta\right)^{t} u(y(t)) d t$. Multiplying both sides by $\left(\beta^{\frac{1}{\lambda}} \delta\right)^{-\eta}$, then adding

$$
\int_{\eta}^{a}\left(\beta^{\frac{1}{\lambda}} \delta\right)^{t-\eta} u(z(t)) d t+\int_{b}^{\eta+\lambda}\left(\beta^{\frac{1}{\lambda}} \delta\right)^{t-\eta} u(z(t)) d t+\beta \int_{\eta+\lambda}^{\infty} \delta^{t-\eta} u(z(t)) d t
$$

to both sides yields:

$$
\begin{gathered}
\int_{\eta}^{a}\left(\beta^{\frac{1}{\lambda}} \delta\right)^{t-\eta} u(z(t)) d t+\int_{a}^{b}\left(\beta^{\frac{1}{\lambda}} \delta\right)^{t-\eta} u(x(t)) d t+\int_{b}^{\eta+\lambda}\left(\beta^{\frac{1}{\lambda}} \delta\right)^{t-\eta} u(z(t)) d t+\beta \int_{\eta+\lambda}^{\infty} \delta^{t-\eta} u(z(t)) d t \\
\geqslant \\
\int_{\eta}^{a}\left(\beta^{\frac{1}{\lambda}} \delta\right)^{t-\eta} u(z(t)) d t+\int_{a}^{b}\left(\beta^{\frac{1}{\lambda}} \delta\right)^{t-\eta} u(y(t)) d t+\int_{b}^{\eta+\lambda}\left(\beta^{\frac{1}{\lambda}} \delta\right)^{t-\eta} u(z(t)) d t+\beta \int_{\eta+\lambda}^{\infty} \delta^{t-\eta} u(z(t)) d t .
\end{gathered}
$$

The above expression is equivalent to $x[a, b) z \gtrsim_{\eta} y[a, b) z$, as required.

It is now shown time-consistency-beyond- $\lambda$-from-now holds. The first preference $x[a, b) z \gtrsim_{\eta} y[a, b) z$, given $\eta \leqslant \lambda \leqslant a \leqslant b$, is equivalent to:

$$
\begin{gathered}
\int_{\eta}^{\lambda}\left(\beta^{\frac{1}{\lambda}} \delta\right)^{t-\eta} u(z(t)) d t+\beta\left(\int_{\lambda}^{a} \delta^{t-\eta} u(z(t)) d t+\int_{a}^{b} \delta^{t-\eta} u(x(t)) d t+\int_{b}^{\infty} \delta^{t-\eta} u(z(t)) d t\right) \\
\geqslant \\
\int_{\eta}^{\lambda}\left(\beta^{\frac{1}{\lambda}} \delta\right)^{t-\eta} u(z(t)) d t+\beta\left(\int_{\lambda}^{a} \delta^{t-\eta} u(z(t)) d t+\int_{a}^{b} \delta^{t-\eta} u(y(t)) d t+\int_{b}^{\infty} \delta^{t-\eta} u(z(t)) d t\right)
\end{gathered}
$$

which holds if and only if $\int_{a}^{b} \delta^{t-\eta} u(x(t)) d t \geqslant \int_{a}^{b} \delta^{t-\eta} u(y(t)) d t$. Multiplying both sides by $\beta \delta^{\eta}$, then adding

$$
\int_{0}^{\lambda}\left(\beta^{\frac{1}{\lambda}} \delta\right)^{t} u(z(t)) d t+\beta\left(\int_{\lambda}^{a} \delta^{t} u(z(t)) d t+\int_{b}^{\infty} \delta^{t} u(z(t)) d t\right)
$$


to both sides yields:

$$
\begin{gathered}
\int_{0}^{\lambda}\left(\beta^{\frac{1}{\lambda}} \delta\right)^{t} u(z(t)) d t+\beta\left(\int_{\lambda}^{a} \delta^{t} u(z(t)) d t+\int_{a}^{b} \delta^{t} u(x(t)) d t+\int_{b}^{\infty} \delta^{t} u(z(t)) d t\right) \\
\geqslant \\
\int_{0}^{\lambda}\left(\beta^{\frac{1}{\lambda}} \delta\right)^{t} u(z(t)) d t+\beta\left(\int_{\lambda}^{a} \delta^{t} u(z(t)) d t+\int_{a}^{b} \delta^{t} u(y(t)) d t+\int_{b}^{\infty} \delta^{t} u(z(t)) d t\right)
\end{gathered}
$$

The above expression is equivalent to $x[a, b) z \gtrsim_{0} y[a, b) z$, as required.

\section{A.2 Proof of Theorem 5.1}

The sufficiency of the axioms for invariant separable discounting is established. The necessity of the axioms involves only simple substitutions. Consider timezero preferences $\succsim_{0} \in \mathcal{R}$. Axioms 1-5 imply, by Corollary 3 of Kopylov (2010), that $\gtrsim_{0}$ over $\mathcal{C}_{0}$ is represented by the following map:

$$
x \mapsto \int_{0}^{\infty} \pi([t, \infty)) u(x(t)) d t, \quad x \in \mathcal{C}_{0},
$$

where $u: X \rightarrow \mathbb{R}$ is a $\succsim_{0}$-increasing utility for outcomes, and $\pi$ is a countably additive measure with $\pi([0, \infty))=1$ and $\pi(t)=0$ for all $t \in T$. In this representation, $\pi$ is unique and $u$ is cardinal.

For all $a<b$, and $x, y \in \mathcal{C}_{0}^{*}$ with $x>y$, the interval monotonicity axiom A3 implies $x[a, b) y>y$. Therefore, $\pi([a, b))>0$ for all $a<b .^{8}$ Now define a discount function $D: T \rightarrow \mathbb{R}$ so that $D(t)=\pi([t, \infty))$ for all $t \in T$. Then $D$ is strictly decreasing. Because $\pi([t, t))=0$ for all $t \in T, D$ is continuous. By construction, $D(0)=1$ and $\lim _{t \rightarrow \infty} D(t)=0$, and the map $x \mapsto \int_{0}^{\infty} D(t) u(x(t)) d t$ represents $\succsim_{0}$ over $\mathcal{C}_{0}$. We write that $\succsim_{0}$ is represented by $(D, u)$.

Now consider $\succsim_{\eta} \in \mathcal{R}$ with $\eta \geqslant 0$. By the same procedure as above, $\gtrsim_{\eta}$ is represented by some $\left(D_{\eta}, u_{\eta}\right)$, with $D_{\eta}: T_{\eta} \rightarrow \mathbb{R}$ unique and $u_{\eta}$ cardinal. Given $\eta$,

\footnotetext{
${ }^{8}$ Kopylov (2010) used a weaker monotonicity than ours, and used stationarity to establish this fact, which also reduced the model to exponential discounting.
} 
define $D_{0, \eta}: T_{\eta} \rightarrow \mathbb{R}$ such that $D_{0, \eta}(t)=D(t-\eta)$ for all $t \in T_{\eta}$. Time invariance asserts that, for all $x, y \in \mathcal{C}_{0}, x \gtrsim_{0} y$ if and only if $x_{\eta} \gtrsim_{\eta} y_{\eta}$. Hence, $\gtrsim_{\eta}$ is represented by $\left(D_{0, \eta}, u\right)$ and, by the uniqueness results above, $D_{0, \eta}=D_{\eta}$ and $u_{\eta}$ can be chosen equal to $u$. Because $\eta$ was arbitrary, every preference $\gtrsim_{\eta} \in \mathcal{R}$ can be represented by $\left(D_{0, \eta}, u\right)$ with $D_{0, \eta}(t)=D(t-\eta)$ for all $t \in T_{\eta}$, and the theorem follows.

\section{A.3 Proof of Theorem 7.4}

The conditions of Theorem 5.1 hold, hence $\mathcal{R}$ is represented by a dynamic model $\mathcal{V}$ such that, for all $V_{\eta} \in \mathcal{V}, V_{\eta}(x)=\int_{\eta}^{\infty} D(t-\eta) u(x(t)) d t$, with $u: X \rightarrow \mathbb{R}$ a $\gtrsim_{\eta}$-increasing utility and $D: T_{\eta} \rightarrow \mathbb{R}$ a strictly decreasing discount function with $D(0)=1$ and $\lim _{s \rightarrow \infty} D(s)=0$. An implication of the definition of timeconsistency-within- $t$-from-now is that, if the condition holds for $\bar{t} \in T$, then it holds for all $t \in[0, \bar{t}]$. Let $[0, \bar{t}]$ be largest interval such that time-consistencywithin- $\bar{t}$-from-now holds. For a given $\eta \in[0, \bar{t}]$, define a function $D_{\eta}:[\eta, \bar{t}] \rightarrow \mathbb{R}$ such that $D_{\eta}(t)=D(\eta) D(t-\eta)$ for all $t \in[\eta, \bar{t}]$. It will be shown that $D$ and $D_{\eta}$ coincide on $[\eta, \bar{t}]$.

Let $x=x[\eta, \bar{t}) z$ and $y=y[\eta, \bar{t}) z$, for some $x, y, z \in \mathcal{C}_{0}$. By the established representation, $x[\eta, \bar{t}) z \gtrsim_{0} y[\eta, \bar{t}) z$ holds if and only if:

$$
\int_{\eta}^{\bar{t}} D(t) u(x(t)) d t \geqslant \int_{\eta}^{\bar{t}} D(t) u(y(t)) d t
$$

By time-consistency-within- $\bar{t}$-from-now, $x[\eta, \bar{t}) z \gtrsim_{0} y[\eta, \bar{t}) z$ if and only if $x[\eta, \bar{t}) z \gtrsim_{\eta}$ $y[\eta, \bar{t}) z$, equivalent to:

$$
\begin{aligned}
& \int_{\eta}^{\bar{t}} D(t-\eta) u(x(t)) d t \geqslant \int_{\eta}^{\bar{t}} D(t-\eta) u(y(t)) d t, \\
& \Leftrightarrow \int_{\eta}^{\bar{t}} D_{\eta}(t) u(x(t)) d t \geqslant \int_{\eta}^{\bar{t}} D_{\eta}(t) u(y(t)) d t .
\end{aligned}
$$


For a given $z \in \mathcal{C}_{0}$, let $\mathcal{C}_{\eta, \bar{t}, z}:=\left\{x \in \mathcal{C}_{0}: x=x[\eta, \bar{t}) z\right\}$. Preferences $\gtrsim_{0}$ over $\mathcal{C}_{\eta, \bar{t}, z}$ are equivalently represented by both $(D, u)$ and $\left(D_{\eta}, u\right)$. The set $\mathcal{C}_{\eta, \bar{t}, z}$, if nonempty, is rich enough to ensure that the uniqueness results of Kopylov's theorem hold. Because $D_{\eta}(0)=1$ and $\lim _{s \rightarrow \infty} D_{\eta}(s)=0$, we have $D=D_{\eta}$ on $[\eta, \bar{t}]$. We chose $\eta$ arbitrarily from $[0, \bar{t}]$, hence $D$ and $D_{\eta}$ coincide on $[0, \bar{t}]$. Equivalently, $D$ satisfies the functional equation: $D(s+t)=D(s) D(t)$ for all $s, t, s+t \in[0, \bar{t}]$. The general, continuous solution gives $D(t)=\alpha \gamma^{t}$ for all $t \in[0, \bar{t}]$ for non-zero $\alpha$ and $\gamma$ (see Aczel and Skof, 2007). The initial condition, $D(0)=1$, gives $\alpha=1$.

If time-consistency-beyond-t-from-now holds for $\bar{t} \in T$, then it holds for all $t \in$ $[\bar{t}, \infty)$. Let $t$ be the smallest $t$ such that time-consistency-beyond- $t$-from-now holds. Similar to above, for a given $\eta \in[\underline{t}, \infty)$, define a function $D_{\eta}:[\underline{t}, \infty) \rightarrow \mathbb{R}$ such that $D_{\eta}(t)=D(\eta) D(t-\eta)$ for all $t \in[\underline{t}, \infty)$. It will be shown that $D$ and $D_{\eta}$ coincide on $[\underline{t}, \infty)$. For all $x, y, z \in \mathcal{C}_{0}, x[\underline{t}, \infty) z \gtrsim_{\eta} y[\underline{t}, \infty) z$ if and only if:

$$
\begin{aligned}
& \int_{\underline{t}}^{\infty} D(t-\eta) u(x(t)) d t \geqslant \int_{\underline{t}}^{\infty} D(t-\eta) u(y(t)) d t, \\
& \Leftrightarrow \int_{\underline{t}}^{\infty} D_{\eta}(t) u(x(t)) d t \geqslant \int_{\underline{t}}^{\infty} D_{\eta}(t) u(y(t)) d t .
\end{aligned}
$$

By time-consistency-beyond- $t$-from-now, $x[\underline{t}, \infty) z \gtrsim_{\eta} y[\underline{t}, \infty) z$ if and only if $x[\underline{t}, \infty) z \succsim_{0}$ $y[\underline{t}, \infty) z$, equivalent to:

$$
\int_{\underline{t}}^{\infty} D(t) u(x(t)) d t \geqslant \int_{\underline{t}}^{\infty} D(t) u(y(t)) d t .
$$

For a given $z \in \mathcal{C}_{0}$, let $\mathcal{C}_{\eta, t, z}:=\left\{x \in \mathcal{C}_{0}: x=x[\underline{t}, \infty) z\right\}$. Preferences $\gtrsim_{0}$ over $C_{\eta, t, z}$ are equivalently represented by both $(D, u)$ and $\left(D_{\eta}, u\right)$, and $C_{\eta, \bar{t}, z}$, if nonempty, is rich enough to ensure that the uniqueness results of Kopylov's theorem hold, hence $D=D_{\eta}$ on $[\underline{t}, \infty]$. Equivalently, $D$ satisfies the functional equation: $D(s+t)=D(s) D(t)$ for all $s, t, s+t \in[\underline{t}, \infty]$. The general, continuous solution gives $D(t)=\beta \delta^{t}$ for all $t \in[\underline{t}, \infty]$ for non-zero, positive $\beta$ and $\delta$. 
It has been shown that $\mathcal{R}$ is represented by a dynamic model $\mathcal{V}$ such that, for all $V_{\eta} \in \mathcal{V}, V_{\eta}(x)=\int_{\eta}^{\infty} D(t-\eta) u(x(t)) d t$, with:

$$
D(t-\eta)= \begin{cases}\gamma^{t-\eta} & \text { if } t-\eta \in[0, \bar{t}], \\ \beta \delta^{t-\eta} & \text { if } t-\eta \in[\underline{t}, \infty) .\end{cases}
$$

Complementary time consistency requires that at least one of time-consistencywithin-t-from-now, or time-consistency-beyond- $t$-from-now, holds for all $t \in T$. Therefore, $[0, \bar{t}] \cup[\underline{t}, \infty)=T$, which requires $\bar{t} \geqslant \underline{t}$. Should these intervals overlap $(\bar{t}>\underline{t})$, there would be an interval $[\underline{t}, \bar{t}]$ such that preferences $\gtrsim_{0}$ for consumption streams, constant outside this interval, are represented by both $\left(\gamma^{t}, u\right)$ and $\left(\beta \delta^{t}, u\right)$, which holds if and only if $\gamma=\delta$ and $\beta=1$ (exponential discounting). Otherwise, we have $\bar{t}=\underline{t}=: \lambda \in(0, \infty)$. In that case, continuity of $D$ requires $\gamma=\beta^{\frac{1}{\lambda}} \delta$, with $0 \leqslant \delta \leqslant 1$, and $0 \leqslant \beta \leqslant \frac{1}{\delta^{\lambda}}$, and continuous quasi-hyperbolic discounting holds.

\section{A.4 Proof of Proposition 8.1}

Assume first that there are no $x, y \in \mathcal{C}_{0}^{*}$ with $x>_{0} y, 0<a<b<c<d<\infty$ and $t>0$, such that $x[a, b) y \sim_{0} x[c, d) y$ and $x[a+t, b+t) y \nsim_{0} x[c+t, d+t) y$. In that case, the stationarity axiom holds, $\mathrm{CQH}$ discounting reduces to exponential discounting, hence $\beta=1$. Now assume instead there there exist $x, y \in \mathcal{C}_{0}^{*}$ with $x>_{0} y, 0<a<b<c<d<\infty$ and $t>0$, such that $x[a, b) y \sim_{0} x[c, d) y$ and $x[a+t, b+t) y \nsim_{0} x[c+t, d+t) y$. It will be shown that, in this case, axiom $8^{*}$ holds if and only if $\beta<1$. CQH reduces to exponential discounting when considering streams that only differ before the switch point $\lambda$ and streams that only differ after the switch point. The assumed violation of stationarity, therefore, reveals that $\lambda$ cannot be earlier than $a$ and cannot be later than $d+t$. That is, $\lambda \in[a, d+t]$. Given $x>_{0} y$, one can find $0<\tilde{a}<\tilde{b}<\tilde{b}+s<a<d+t<\tilde{c}<\tilde{d}<\infty$ such that $x[\tilde{a}, \tilde{b}) y \sim_{0} x[\tilde{c}, \tilde{d}) y$. By construction, $\tilde{b}<\lambda<\tilde{c}$. Substituting CQH discounting 
and cancelling common terms, this indifference is equivalent to:

$$
\int_{\tilde{a}}^{\tilde{b}}\left(\beta^{\frac{1}{\lambda}} \delta\right)^{t} u(x) d t+\beta \int_{\tilde{c}}^{\tilde{d}} \delta^{t} u(y) d t=\int_{\tilde{a}}^{\tilde{b}}\left(\beta^{\frac{1}{\lambda}} \delta\right)^{t} u(y) d t+\beta \int_{\tilde{c}}^{\tilde{d}} \delta^{t} u(x) d t .
$$

Rearranging and dividing by $u(x)-u(y)>0$ gives:

$$
\int_{\tilde{a}}^{\tilde{b}}\left(\beta^{\frac{1}{\lambda}} \delta\right)^{t} d t=\beta \int_{\tilde{c}}^{\tilde{d}} \delta^{t} d t
$$

By axiom $8^{*}$, the above indifference holds only if, for $s>0$ such that $\tilde{b}+s<a$, we have $x[\tilde{a}+s, \tilde{b}+s) y \prec_{0} x[\tilde{c}+s, \tilde{d}+s) y$. Substituting $\mathrm{CQH}$ discounting and cancelling common terms as above yields:

$$
\begin{gathered}
\int_{\tilde{a}+s}^{\tilde{b}+s}\left(\beta^{\frac{1}{\lambda}} \delta\right)^{t} d t<\beta \int_{\tilde{c}+s}^{\tilde{d}+s} \delta^{t} d t, \\
\Leftrightarrow\left(\beta^{\frac{1}{\lambda}} \delta\right)^{s} \int_{\tilde{a}}^{\tilde{b}}\left(\beta^{\frac{1}{\lambda}} \delta\right)^{t} d t<\delta^{s} \beta \int_{\tilde{c}}^{\tilde{d}} \delta^{t} d t,
\end{gathered}
$$

Taken together, equation 6 and inequality 7 hold only if:

$$
\left(\beta^{\frac{1}{\lambda}} \delta\right)^{s}<\delta^{s} \quad \Leftrightarrow \quad \beta^{\frac{1}{\lambda}}<1 .
$$

Because $0<\lambda<\infty$, it follows that $\beta<1$. 